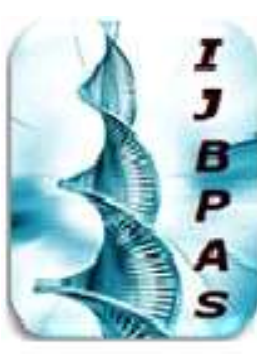

International Journal of Biology, Pharmacy and Allied Seiences (IJBPAS) 'A Bridge Betusen Caboratory and QRendo'

WwW.ijbpas.com

\title{
A REVIEW ON PHARMACEUTICAL CO-CRYSTALS
}

\section{DONGARE AM*, GADHAVE MV, DHOBALE SM, JADHAV SL, GAIKWAD DD}

Vishal Junnar Seva Mandal's Vishal Institute of Pharmaceutical Education and Research, Ale, Tal. Junnar, Dist. Pune, M.S., India

*Corresponding Author: E Mail: agastidongare2015@gmail.com

Received 27 ${ }^{\text {th }}$ April 2021; Revised $25^{\text {th }}$ June 2021; Accepted $1^{\text {st }}$ Aug. 2021; Available online $1^{\text {st }}$ Oct. 2021

https://doi.org/10.31032/IJBPAS/2021/10.10.1037

ABSTRACT

According to the BCS classification system class 2 drug have low solubility and high permeability. In the present study it was, planned to review the improved solubility of BCS class 2 drugs by preventing its dihydrate formation, by using co-crystal formation approach. It is widely accepted method to improve physical properties of BCS class 2 drug especially solubility.Co-crystal are prepared by using co-crystal former because it contains many hydroxyl groups and thus has potential to form hydrogen bonds with other components.

Keywords: Co-crystal, co-former, solubility

\section{INTRODUCTION}

Due to the introduction of highly advanced tools in drug discovery pipeline like high trough put screening (HTS), a vast number of a hydrophobic drug molecule with high molecular weight are developing low aqueous solubility of hydrophobic drug having less absorption, poor bioavailability, and develop a leading problem in product development regarding route of administration, active pharmaceutical ingredient need to dissolve or to be solubilized in an aqueous environment at least for the same time of drug in the blood to produced its therapeutic action. Limited aqueous solubility drug often need to give more amount of doses so they can achieve optimum plasma concentration after oral drug administration [1]. The solubility related problems increasing day by day, and they are more prominent in new chemical 
entities. A considerable number of first pass metabolism, drug aqueous commercially marketed compound solubility and dissolution rate. The (Approximately 40\%) and drug under developmental pipeline (Approximately $80 \%$ ) facing solubility related issue [2]. Challenges associated with poor aqueous solubility may be seen in the early phase of drug development processes, and thus there are increasing efforts to link drug discovery and development process to formulation strategies [3].

\subsection{Importance of solubility in product development}

The oral route of drug administration is the most preferable and most suitable route of administration. As this route have its own advantages like more patient compliance, less sterility restriction, less manufacturing cost, and most prominently flexible in designing in the dosage form though, the most vital task with the fabrication of oral dosage form lies in there less bioavailability. The oral bioavailability depends on the number of factors like drug permeability, enhancement of solubility and dissolution rate is one of the most thought-provoking features of the development of dosage forms, particularly for the oral dosage form various approaches are reported scientific literature to improve the solubility of limited aqueous soluble drug [4].

\subsection{Formulation approaches for improvement of solubility}

Numerous formulation techniques employed for the enhancement of clinical efficiency of poor water soluble drug. According to the biopharmaceutical classification system (BCS) of drugs, the drugs are categorized into four major categories based on their solubility and permeability behavior. They are as follows, Class I- high solubility and high permeability, class II- low solubility and high permeability, class III- high solubility and low permeability, and class IV- low solubility and low permeability [5].

Table 1.1 gives a brief idea about various formulation approaches employed for the manufacturing of the dosage form of various BCS classes of drugs

\begin{tabular}{|c|c|}
\hline $\begin{array}{c}\text { Class- I High solubility and high permeability } \\
\text { Immediate release and sustained release }\end{array}$ & $\begin{array}{c}\text { Class- II Low solubility and high permeability } \\
\text { Modification of drug crystal habits, particle size reduction, } \\
\text { solid dispersion, complexation, salt formation. }\end{array}$ \\
\hline $\begin{array}{c}\text { Class- III High solubility and low permeability } \\
\text { Immediate release formulation with an absorption } \\
\text { enhancer. }\end{array}$ & $\begin{array}{c}\text { Class- IV Low solubility and low permeability } \\
\text { Class II approaches or joint approaches for class II along } \\
\text { with absorption enhancer. }\end{array}$ \\
\hline
\end{tabular}

Table 1.1 Feasible formulation approaches The solubility improvement approaches are employed for the manufacturing of dosage broadly classified into two categories i.e. form of BCS classes of drug. 
physical and chemical approaches of solubility enhancement.

\subsubsection{Physical approaches to solubility enhancement}

The physical approach does not play with the structure of the drug molecule [6]. In physical method, a drug molecule physically modulated by mean of any of the excipient added to it, such polymer for solid dispersion, or sometime particle size of drug is reduced to micron level to improve its solubility. The physical method includes particle size reduction, modification of drug crystal habits, drug dispersed into the hydrophilic carrier, complexation with cyclodextrin, and solubilization by using a surfactant molecule [7].

\subsubsection{Particle size reduction}

The solubility of drug is often intrinsically related to drug particle size; as a particle becomes smaller, the surface area to volume ratio increases. The larger surface area allows greater interaction with the solvent which causes an increase in solubility [8]. Conventional methods of particle size reduction, such as comminution and spray drying, rely upon mechanical stress to disaggregate the active compound. Particle size reduction is thus permitting an efficient, reproducible, and economic means of solubility enhancement. However, the mechanical forces inherent to comminution, such as milling and grinding, often impart significant amounts of physical stress upon the drug product which may induce degradation. The thermal stress which may occur during comminution and spray drying is also a concern when processing thermosensitive or unstable active compounds. Using traditional approaches for nearly insoluble drugs may not be able to enhance the solubility up to desired level. Micronization is another conventional technique for the particle size reduction [9]. Micronization increases the dissolution rate of drugs through increased surface area, it does not increase equilibrium solubility. Decreasing the particle size of these drugs, which cause increase in surface area, improve their rate of dissolution. Micronization of drugs is done by milling techniques using jet mill, rotor stator colloid mills and so forth micronization is not suitable for drugs having a high dose number because it does not change the saturation solubility of the drug [10].

\subsubsection{Solid dispersion}

The concept of solid dispersions was originally proposed by Sekiguchi and Obi, who investigated the generation and dissolution performance of eutectic melts of a sulfonamide drug and a water-soluble 
carrier in the early 1960s [11]. Solid dispersions represent a useful pharmaceutical technique for increasing the dissolution, absorption, and therapeutic efficacy of drugs in dosage forms [12]. The term solid dispersion refers to a group of solid products consisting of at least two different components, generally a hydrophilic matrix and a hydrophobic drug. The most commonly used hydrophilic carriers for solid dispersions include polyvinylpyrrolidone (Povidone, PVP), polyethylene glycols (PEGs), PlasdoneS630. Surfactants like Tween-80, docusate sodium, Myrj-52, Pluronic-F68, and sodium lauryl sulphate (SLS) also find a place in the formulation of solid dispersion [14].

\subsubsection{Self-emulsifying drug delivery system}

The self-emulsifying drug delivery system is a lipid-based formulation that enhances the solubility and dissolution rate of the combined hydrophobic drug. The delivery system contains a mixture of oil (natural or synthetic lipids, surfactant, co-surfactant and co-solvent) the drug delivery system classically possesses self-dispersing ability as well as the capability to form $\mathrm{O} / \mathrm{W}$ emulsion or micro emulsion after slight agitation. The disadvantages is of the system are chemical instability of drugs, and some high use surfactant may cause gastro intestinal toxicity after oral administration [15].

\subsubsection{Modification of drug crystal habits}

This method involves the formation of the noncovalent derivative of a drug molecule by incorporating one or more suitable GRAS listed compounds. Different types of formulation are obtained during modification of drugs crystal habit like co-crystal, eutectics systems, and solid solutions [16]. The invention provides methods of modifying the crystal habit of a compound without altering the crystal structure of the compound through a controlled precipitation technique in the presence of a crystal growth inhibitor as well as the crystallized compounds formed by these methods. Using these methods, the crystal habit of the compound may be modified from acicular to bipyramidal. The modification in crystal habit [17] is attributable to a preferential adsorption mechanism of the crystal growth inhibitor to a fast growing crystal face of the compound. Powder flow properties of the crystallized product are significantly enhanced with the habit modification. This selective crystal habit modification using a crystal growth inhibitor provides a strategy to circumvent the manufacturing difficulties associated with acicular crystal habits [18]. 


\subsubsection{Chemical approaches to solubility enhancement}

In this approach, the chemical structure of active pharmaceutical ingredient is modified for the alteration of its biopharmaceutical properties. This method includes the formation of a new chemical bond with same another molecule like the salt formation of prodrug formation [19].

\subsubsection{Salt formation}

Salt formation is a neutralization reaction between acids and bases. Salt formation is used for drugs that can be ionized so that solubility increases. Salt is formed by the transfer of protons from acids to bases [20]. Ionic bonds from salts can form and are stable if the difference in the $\mathrm{pKa}$ (ionization constant) between acids and bases $(\Delta \mathrm{pKa})$ is $>3$ [21]. Through this reaction, salt formation requires a counter ion, stoichiometric molar ratio, and a suitable solvent. The selection of the proper salt structure of active drug ingredients must be efficient and rational, as the salt selection can influence the preformulation evaluation of drugs. Many criteria must be considered in the formation of salt compounds: the route of drug administration, biological factors, $\mathrm{pKa}$, biopharmaceutical factors, ionic factors, and organic solvents selected [21].

\subsubsection{Prodrug formation}

The prodrug is a biologically inactive form of an active drug molecule that usually required to enzymatic or chemical biotransformation within the body to release its active form in this method, a drug molecule is covalently joined with pro moiety. The disadvantages of prodrug formation is a metabolite formed during biotransformation is sometime produce toxicity [22].

\subsubsection{Hydrotrophy}

Hydrotrophy is a solubilization process, whereby addition of a large amount of second solute, the hydrotropic agent results in an increase in the aqueous solubility of first solute. Hydrotropic agents are ionic organic salts, consists of alkali metal salts of various organic acids. Additives or salts that increase solubility in given solvent are said to "salt in" the solute [23] and those salts that decrease solubility "salt out" the solute. Several salts with large anions or cations that are themselves very soluble in water result in "salting in" of non electrolytes called "hydrotropic salts"; a phenomenon known as "hydrotropism." Hydrotrophy designate the increase in solubility in water due to the presence of large amount of additives. The mechanism by which it improves solubility is more closely related to complexation involving a weak interaction between the hydrotropic agents like sodium benzoate, 
sodium acetate, sodium alginate, urea, and the poorly soluble drugs [24].

\subsubsection{Complexation with cyclodextrin}

Basically, in this approach, drug form with $\beta$ cyclodextrin through host-guest interaction. Cyclodextrins are structurally related to cyclic oligosaccharide having a lipophilic cavity and hydrophilic external surface [25]. The inclusion complex are formed by the insertion of lipophilic drug into the lipophilic cavity of the cyclodextrin molecule through noncovalent interaction [26].

\subsection{Pharmaceutical co-crystals}

The pharmaceutical co-crystal are structural homologs to crystalline material composed of two or more neutral molecules present indefinite stochiometric ratios. In co-crystal, drug and coformer are assembled by noncovalent interaction like hydrogen bonding, pi-pi interaction, van der waals interaction [27]. The first co-crystal was synthesized by scientist Freidrichwohler is quinhydron co-crystal by using benzoquinone and hydroquinone at 1:1 stochiometric ratio. The co-crystal can give an apportunity to alter drug physiological properties like solubility, stability, melting point, hygroscopicity, and mechanical properties like flowability compressibility. As well, they can improve pharmacological properties like bioavailability and permeability [28]. The charge transfer is one of the commonly seen interaction in the synthesis of organic co-crystal. Hydrogen bond play a most significant role in the formation of organic co-crystal. Some of nocovalentinteractions are also dominating in the co-crystal self-assembly process. They also impact direct structural stability as well as physiological properties in co-crystals [29].

\subsubsection{Components of pharmaceutical co- crystal}

The co-crystal contains two main constituents they are as follows

- Coformers or another drug moleculethese are non-toxic, and GRAS listed compound having the capability to form co-crystal with drug.

- Active pharmaceutical ingredientstherapeutic molecule having physiochemical problems like low solubility, instability, poor flow properties, less permeability, etc.

These two components get joined together with the help of noncovalent interaction like hydrogen bonding or van der waals interaction. The synthons are the structural units, in which two molecules get assembled or joined with intermolecular interaction [30]. There are two types of synthons available. 
A- Homosynthons- In this type of synthon, the structural unit within supramolecule can be formed within the same functional group are called homosynthon. The following Figure 1.1 summarises example of homosynthones.

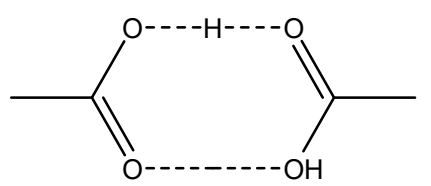

Acid-acid diamer

Figure 1.1
B- Heterosynthons- In this type of synthon, thestructural units within supramolecule can be formed within the different functional group. The following Figure $\mathbf{1 . 2}$ explains examples of heterosynthons.

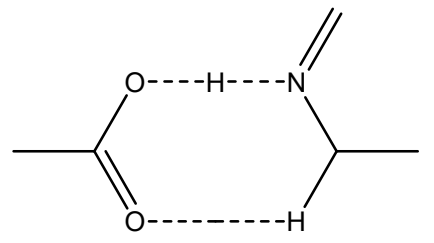

Acid-pyrimidine diamer

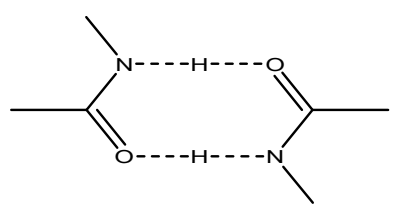

Amide-amide diamer

Figure 1.2

\subsubsection{Advantages of pharmaceutical co-} crystal

Following are some advantages offered by co-crystals,

* No by-product formation takes place during the synthesis of co-crystal.

* The molecular structure of drug remains unchanged in the synthesis of co-crystal.

High yielding techniques.

Synthesis of co-crystals is patentable innovation.

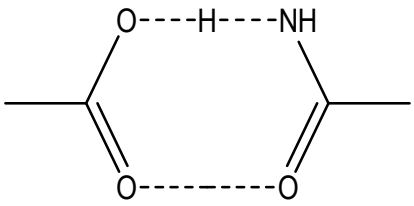

Acid-amide diamer

Almost all type of molecule can form a co-crystal.

\subsection{Coformer selection stratergies}

The selection of suitable coformer molecule for the synthesis of co-crystal is the most challenging and tedious task in the entire cocrystal manufacturing process. There are different coformer prediction tools for synthesis of co-crystal are available like Hansen solubility parameter, pka etc.

\subsubsection{Hansen solubility parameter}

Hansen solubility parameter is another important approach used to measure the 
miscibility of drug and coformers used for co-crystal systems. The miscibility of the components in the solid state could predict the co-crystal formation. The synthesis of cocrystals success rate was improved by using the components which have similar miscibility. It was demonstrated that the two components should be miscible if total HSPs differencewas $\quad<7 \mathrm{MP}^{0.5}, \quad$ otherwise immiscible. Another method estimates the miscibility of two components if the difference is $\leq 5$ MP0.5 between two substances which are supposed to be cocrystal formation [31].

\subsection{2 $\Delta$ pKa rule}

Cocrystals or salts formation can be predicted by proton transfer between acid and base. The formation of salts or cocrystals can be predicted by determining the $\Delta \mathrm{pKa}=[\mathrm{pKa}$ (base)-pKa (acid)]. It is generally accepted that proton transfer will occur from acid to base if the difference in the $\mathrm{pKa}$ values is greater than 2 or 3 . A smaller $\Delta \mathrm{pKa}$ value (less than 0 ) indicates the formation of cocrystals whereas higher value (more than 2 or 3 ) indicates the formation of salts [32]. $\Delta \mathrm{pKa}$ rule was validated and quantified by studying 6465 cocrystals from CSD and explained a linear relationship between $\Delta \mathrm{pKa}$ value and possibility of proton transfer between acid-base pair. It was analysed that a non-ionized complex should be formed when the value of $\Delta \mathrm{pKa}<-1$; an ionized complex is formed when the value of $\Delta \mathrm{pKa}<4$ and the possibility of formation of ionized complex increase by $17 \%$ by increase in $\Delta \mathrm{pKa}$ by one unit from $10 \%$ at $\Delta \mathrm{pKa}=-1$ to $95 \%$ at $\Delta \mathrm{pKa}=4$. By determining the $\Delta \mathrm{pKa}$ value, the possibility of formation of cocrystals and salts can be determined. This is a simple and less time-consuming method for the preparation of co-crystals [33].

\subsubsection{Fabian's method}

Different sets of reliable co-crystal forming structures were extracted from the CSD and the molecular descriptors (single atom, bond and group counts, hydrogen bond donor and acceptor counts, size and shape, surface area and molecular electrostatic) were calculated for each molecule. On the basis of calculated molecular properties, the database described pairs of molecules that were able to form cocrystals. The strongest descriptor correlation was related to the shape and polarity of co-crystal formers [34].

\subsection{Properties altered by co-crystalisation}

The alteration in molecular structure assemblies may lead to changes in physical properties. The expected changes in physiochemical properties would occur such as solubility, melting point, stability and other mechanical properties. There are some 
example which demonstrate the change in physiochemical properties [35].

\subsubsection{Melting point}

Melting point is the physical property of solids, which is used to determine the purity of the product with sharp melts and narrow ranges. High melting point demonstrates the thermodynamically stability of the new materials i.e. thermal stability of an API can be increased by selecting the coformer with higher melting point. Cocrystals with low melting points can also be beneficial when dealing with thermolabile drugs [36]. The most commonly used techniques for determination of melting point and thermal analysis are differential scanning calorimetry (DSC) and thermal gravimetric analysis (TGA). Melting point of pharmaceutical cocrystals can be tailored by judicious selection of the coformers. Melting point of about 50 cocrystals was analysed and the results showed that $51 \%$ cocrystals showed melting points between API and coformers, while $39 \%$ cocrystals have melting point lower than either API or coformer, $6 \%$ have high melting point than both API and coformer, and $4 \%$ had same about API or conformer [37].

\subsubsection{Solubility}

Solubility is an important parameter to investigate the formulations of poorly soluble drugs. Many approaches have been used to improve the solubility of drugs such as salt formation, solid dispersion, particle size reduction, and so on, amongst which cocrystallization has been used by several researchers. Solubility of antifungal drug ketoconazole was increased 53 and 100 times by synthesizing salts and cocrystals respectively as compared to ketoconazole. Thus, higher solubility of drug was obtained by cocrystals as compared to salt formation [38].

\subsubsection{Stability}

Stability study is extremely important during the development of new dosage formulation. During development of pharmaceutical cocrystals several stability studies should be performed such as relative humidity stress, chemical stability, thermal stability, solution stability and photostability study. In relative humidity stress, automated water sorption/desorption studies are performed to determine the effect of water on the formulation. Several researchers studied the behaviour of cocrystals under relative humidity stress conditions. Cocrystals of glutaric acid and 2-[4-(4-chloro-2fluorphenoxy)phenyl]pyrimidine4carboxamide showed $0.08 \%$ moisture at high 95\% RH and cocrystals were found stable at different conditions [39]. 


\subsubsection{Bioavailability}

Bioavailability is defined as the rate and extent of pure drug that reaches into systemic circulation. Low oral bioavailability of APIs is a major challenge during the development of new formulations [40]. Crystal engineering is mainly used to design and synthesize the pharmaceutical cocrystals with enhanced aqueous solubility and oral bioavailability. Pharmacokinetics study of apixaban-oxalic acid cocrystals in beagle dogs showed that oral bioavailability was increased by 2.7 times as compared to pure drug. Oral bioavailability of baicalein was increased by formation of cocrystals with nicotinamide and showed that cocrystals had 2.49 times higher peak plasma concentration (Cmax) and 2.80 times higher area under the curve (AUC) as compared to pure drug in rats. Meloxicam cocrystals with aspirin exhibited better oral bioavailability as compared to pure drug and showed 12 times faster onset of action than pure drug in rats. Cocrystals of 6-mercaptopurine (a BCS Class-II drug) showed higher oral bioavailability i.e. $168.7 \%$ than pure drug in rats [41].

\subsection{Method for the preparation of cocrystals}

Till date, different methods have been reported for the preparation of cocrystals by the researchers. Few traditional methods based on the solution and grinding were reported for the synthesis of cocrystals [42]. A suitable type of solvent is used in solution method for the preparation of cocrystals. Different types of solution methods such as solvent evaporation, solution crystallization technique, antisolvent addition, slurry conversion method and reaction crystallization method are discussed with suitable examples. Grinding methods are of two types: neat grinding and solvent drop grinding. Some newly emerging methods used for the formation of cocrystals are ultrasound assisted solution method, supercritical fluid atomization technique, spray drying technique, hot melt extrusion technique [43].

\subsubsection{Solvent evaporation method}

In solvent evaporation method, for both API and coformer are dissolved in a suitable solvent and the solution is allowed to evaporate the solvent slowly. During dissolution, the functional groups in the drug and conformer interact with each other and form hydrogen bonds. This is most commonly used method for the preparation of cocrystals by researchers [44].

\subsubsection{Grinding method}

Grinding methods have been widely used for the for the co-crystal formation over the past 
few years and found to be superior than other methods (solution or melt). Grinding techniques are of two types: neat or dry grinding and wet grinding. In dry grinding, drug and coformer are mixed together in a stoichiometric ratio and ground them by using either mortar and pestle or ball mill. Wet Grinding was performed in a similar manner that of neat grinding by addition of some drops of solvent in the mixture [45].

\subsubsection{Ultrasound assisted solution cocrystallization}

Sonochemical method has been developed for the preparation of cocrystals of very small size i.e. for preparation of nanocrystals. In this method, API and cocrystal former are dissolved together in a solvent and the solution is kept in a sonoreactor to form the solution turbid. Cold water is supplied during the sonication to maintain the constant temperature of sonicator and prevent fragmentation. The solution is kept overnight for drying. Pure cocrystals were obtained by this method and the purity of cocrystals can be assessed by using X-ray diffraction study [46].

\subsubsection{Supercritical fluid atomization technique}

In supercritical atomization technique, the drug and coformers are mixed with each other by using high pressurized supercritical fluid i.e. CO2. Cocrystals are prepared by atomizing this solution with the help of atomizer. In supercritical antisolvent (SAS) method, the cocrystals are prepared from solution by the antisolvent effect of supercritical fluid [47].

\subsubsection{Spray drying technique}

In spray drying process, cocrystals are prepared by spraying the solution or suspension of drug and coformer with hot air stream to evaporate the solvent. This is the most preferred technology because this is a fast, continuous, and one-step process. Thus, spray drying process will offer a unique environment for the preparation and scale-up of cocrystals [48].

\subsubsection{Hot melt extrusion technique}

In hot melt extrusion technique, the cocrystals are prepared by heating the drug and coformers with intense mixing which improved the surface contacts without use of solvent. The limitations of this method include both coformer and API should be miscible in molten form and not used for thermolabile drugs.

\subsection{Characterization and evaluation of co- crystal}

The ultimate goal of co-crystal screen is to discover a solid form of an API with improve physical properties. In some cases co-crystal formation is readily apparent from the 
resulting physical properties of the new material.

\subsubsection{Fourier transforms infrared spectroscopy}

FTIR spectroscopy is used to predict the intermolecular interactions and compatibility study between drug and coformers. This technique is widely used to predict the chemical conformation of compounds. Aakeroy et al. used FTIR to distinguish the cocrystals from salts by evaluating the carboxylic acid involvement in the hydrogen bond formation. Pure drug, coformer, physical mixture and cocrystals are analysed by FTIR in the range of $400-4000 \mathrm{~cm}-1$. FTIR study is also used along with other techniques such as DSC or XRD for the screening of the cocrystals [49].

\subsubsection{Differential scanning calorimetry}

DSC has been used for screening of cocrystal formation. Screening of cocrystals formation can be determined by the presence of exothermic peak followed by endothermic peak in DSC spectra. The presence of these peaks in the physical mixture of components indicates the possibility of formation of cocrystals. Pure drug, coformer, physical mixture and cocrystals were weighed out (1.5-2.5 mg) in aluminium pans and analysed with heating rates of $5-30^{\circ}$ using similar empty pan as a reference. The nitrogen gas with flow rate $50 \mathrm{ml} / \mathrm{min}$ maintained the inert atmosphere. Melting point, glass transition temperature, polymorphic nature, heat of fusion, endothermic or exothermic behaviour can be determined by using DSC.

\subsubsection{X-ray diffraction}

PXRD is commonly used for screening and determination of cocrystalstructure. The PXRD patterns obtained from diffractometer were compared to each other for analysing the structure of cocrystals. The different PXRD pattern of cocrystals from their components is the indication of cocrystals formation. Crystal structure of solids at atomic level cocrystals is determined by using single crystal X-ray diffraction (SXRD). The major problem related to this technique is that generally single cocrystal cannot be produced which is suitable for SXRD analysis. Scanning electron microscope is the instrument used to determine the particle size and morphological analysis of cocrystals. A high energy electron beams scan the atoms that provide the information about the sample surface's topography [50].

\subsubsection{In vitro release of co-crystal}

Dissolution study is used to determine the amount of drug release with time in dissolution medium and predict the in vivo performance of the formulation. Dissolution 
studies for the cocrystals can be performed with the help of the dissolution apparatus. The dissolution studies for the cocrystals can be done within the suitable dissolution medium described in drug protocol of referred pharmacopoeia. The drug samples can be collected in the suitable quantity at predetermined time interval and can be examined with the help of suitable means like HPLC or UV.

\subsubsection{Solubility}

Solubility study can be assessed by Higuchi and Connors method for solubility determination. The solubility of pure drug, physical mixture and cocrystals can be determined in water or suitable medium given in the referred pharmacopoeia. Drug sample and medium should be added in a conical flask, and should be shaken for $24 \mathrm{~h}$ at room temperature on rotary flask shaker. The entire samples should be protected from light by wrapping the flask by aluminium foil if the drug is sensitive to light. After $24 \mathrm{~h}$ samples are filtered through Whatman filter paper and aliquots are

suitably diluted and assayed by HPLC or UV at suitable wavelength.

\section{CONCLUSION}

From current review, it can be concluded that improvement of solubility of BCS class 2 drugs by preventing its dihydrate formation, by using co-crystal formation approach is possible. It is widely accepted method to improve physical properties of BCS class 2 drug especially solubility. Co-crystal are prepared by using co-crystal former because it contains many hydroxyl groups and thus has potential to form hydrogen bonds with other components.

\section{REFERENCES}

[1] Savjani KT, Gajjar AK, Savjani JK. Drug Solubility: Importance and Enhancement Techniques. ISRN Pharm. 2012 Jul 5;2012:1-10.

[2] Karagianni A, Kachrimanis K, Nikolakakis I. Co-Amorphous Solid Dispersions for Solubility and Absorption Improvement of Drugs: Composition, Preparation, Characterization and Formulations for Oral Delivery. Pharmaceutics. 2018 Jul 19;10(3):98.

[3] Jindal k. review on solubility: a mandatory tool for pharmaceuticals. Int Res J Pharm. 2017 Dec 19;8(11):11-5.

[4] Sathisaran I, Dalvi S. Engineering Cocrystals of Poorly Water-Soluble Drugs to Enhance Dissolution in Aqueous Medium. Pharmaceutics. 2018 Jul 31;10(3):108. 
[5] Chaudhari S, Nikam SA, Khatri N, Wakde S. co-crystals: a review. j drug DelivTher. 2018 Dec 15; 8(6-s):350 8.

[6] Kamalakkannan V, Puratchikody A, Masilamani K, Senthilnathan B. Available online through www.jpronline.info Solubility enhancement of poorly soluble drugs by solid dispersion technique - A review. J Pharm Res. 2010;(9):9.

[7] Ogawa N, Hiramatsu T, Suzuki R, Okamoto R, Shibagaki K, Fujita K, et al. Improvement in the water solubility of drugs with a solid dispersion system by spray drying and hot-melt extrusion with using the amphiphilic polyvinyl caprolactampolyvinyl acetate-polyethylene glycol graft copolymer and d-mannitol. Eur J Pharm Sci. 2018 Jan;111:205-14.

[8] Kumar R, Siril PF. Enhancing the Solubility of Fenofibrate by Nanocrystal Formation and Encapsulation. AAPS PharmSciTech. 2018 Jan;19(1):284-92.

[9] Newa M, Bhandari KH, Oh DH, Kim YR, Sung JH, Kim JO, et al. Enhanced dissolution of ibuprofen using solid dispersion with poloxamer
407. Arch Pharm Res. 2008

Nov;31(11):1497-507.

[10] Neidle S, Thurston DE. Chemical approaches to the discovery and development of cancer therapies. Nat Rev Cancer. 2005 Apr;5(4):285-96.

[11] Serajuddin ATM. Salt formation to improve drug solubility. Adv Drug Deliv Rev. 2007 Jul;59(7):603-16.

[12] Mesallati H, Umerska A, Paluch KJ, Tajber L. Amorphous Polymeric Drug Salts as Ionic Solid Dispersion Forms of Ciprofloxacin. Mol Pharm. 2017 Jul 3;14(7):2209-23.

[13] Mukherjee A, Rogers RD, Myerson AS. Cocrystal formation by ionic liquid-assisted grinding: case study with cocrystals of caffeine. CrystEngComm. 2018;20(27):381721.

[14] Nidhi K, Indrajeet S, Khushboo M, Gauri K, Sen DJ. hydrotropy: a promising tool for solubility enhancement: a review. 2011;8.

[15] Hamada H, Ishihara K, Masuoka N, Mikuni K, Nakajima N. Enhancement of water-solubility and bioactivity of paclitaxel using modified cyclodextrins. J 
BiosciBioeng.

2006

Oct;102(4):369-71.

[16] Loftsson T, Duchene D. Cyclodextrins and their pharmaceutical applications. Int J Pharm. 2007 Feb 1;329(1-2):1-11.

[17] Kuminek G, Cao F, Bahia de Oliveira da Rocha A, Gonçalves Cardoso S, Rodríguez-Hornedo N. Cocrystals to facilitate delivery of poorly soluble compounds beyondrule-of-5. Adv Drug Deliv Rev. 2016 Jun;101:143-66.

[18] Bolla G, Nangia A. Pharmaceutical cocrystals: walking the talk. ChemCommun. 2016;52(54):834260.

[19] Wang $X$, Du S, Zhang R, Jia X, Yang T, Zhang X. Drug-drug cocrystals: Opportunities and challenges. Asian J Pharm Sci. 2020 Jul;S181808762030427X.

[20] Desiraju

GR.

SupramolecularSynthons in Crystal Engineering-A New Organic Synthesis. AngewChemInt Ed Engl. 1995 Nov 17;34(21):2311-27.

[21] Ghazwani M, Begum MY, Alam P, Alqarni MH, Yusufoglu HS, Shakeel F. Solubility Determination, Hansen Solubility Parameters and
Thermodynamic Evaluation of Thymoquinone in (Isopropanol + Water) Compositions. Molecules. 2021 May 26;26(11):3195.

[22] Serrano DR, O'Connell P, Paluch KJ, Walsh D, Healy AM.Cocrystal habit engineering to improve drug dissolution and alter derived powder properties. J Pharm Pharmacol. 2016 May 25;68(5):665-77.

[23] Bavishi DD, Borkhataria $\mathrm{CH}$. Spring and parachute: How cocrystals enhance solubility. ProgCryst Growth Charact Mater. 2016 Sep;62(3):1-8.

[24] Kumar S, Nanda A. Pharmaceutical Cocrystals: An Overview. Indian J Pharm Sci [Internet]. 2017 [cited 2021 Aug 13];79(6). Available from:

http://www.ijpsonline.com/articles/p harmaceutical-cocrystals-anoverview-3400.html

[25] Nikam VJ, Patil SB. Pharmaceutical cocrystals of nebivolol hydrochloride with enhanced solubility. J Cryst Growth. 2020 Mar;534:125488.

[26] Rama V, Vidavulur S, Tadikonda PV, Rajana N, Mittapalli S. Novel cocrystals of brexpiprazole with 
improved solubility. J Cryst Growth. 2020 Dec;551:125910.

[27] Hazarikajnr, deb p. formulation evaluation and optimization of immediate release tablet of aceclofenac by direct compression method. Int J Curr Pharm Res. 2017 May 5;9(3):118.

[28] Sekar V, Chellan VR. Immediate Release Tablets of Telmisartan Using SuperdisintegrantFormulation, Evaluation and Stability Studies.Chem Pharm Bull (Tokyo). 2008;56(4):575-7.

[29] Garudkar AB, Bhosale DAV, Puttewar TY, Patil dry. design, development and evaluation of immediate release tablet of ibuprofen solid dispersion. Int $\mathrm{J}$ Curr Res. 2016;08:14.

[30] Kharroubi AT. Diabetes mellitus: The epidemic of the century. World J Diabetes. 2015;6(6):850.

[31] Shete A, Murthy S, Korpale S, Yadav A, Sajane S, Sakhare S, et al. Cocrystals of itraconazole with amino acids: Screening, synthesis, solid state characterization, in vitro drug release and antifungal activity. J Drug Deliv Sci Technol. 2015 Aug;28:46-55.
[32] Zhou Z, Li W, Sun W-J, Lu T, Tong HHY, Sun CC, et al. Resveratrol cocrystals with enhanced solubility and tabletability. Int J Pharm. 2016 Jul;509(1-2):391-9.

[33] Kulla H, Greiser S, Benemann S, Rademann K, Emmerling F. In Situ Investigation of a Self-Accelerated Cocrystal Formation by Grinding Pyrazinamide with Oxalic Acid. Molecules. 2016 Jul 14;21(7):917.

[34] Dhat S, Pund S, Kokare C, Sharma P, Shrivastava B. Mechanistic investigation of biopharmaceutic and pharmacokinetic characteristics of surface engineering of satranidazole nanocrystals. Eur J Pharm Biopharm. 2016 Mar;100:109-18.

[35] Cao F, Amidon GL, RodriguezHornedo N, Amidon GE. Mechanistic Analysis of Cocrystal Dissolution as a Function of $\mathrm{pH}$ and Micellar Solubilization. Mol Pharm. 2016 Mar 7;13(3):1030-46.

[36] do Amaral LH, do Carmo FA, Amaro MI, de Sousa VP, da Silva LCRP, de Almeida GS, et al. Development and Characterization of Dapsone Cocrystal Prepared by Scalable Production Methods. 
AAPS PharmSciTech. 2018 Aug;19(6):2687-99.

[37] Gurav NP, Dandagi PM, Gadad AP, Masthiholimath VS. Solubility Enhancement of Satranidazole Using Self. Indian J Pharm Educ Res. 2016;50(3):8.

[38] Pawar SR, Barhate SD. Solubility enhancement (Solid Dispersions) novel boon to increase bioavailability. J Drug Deliv Ther. 2019 Mar 22;9(2):583-90.

[39] Garudkar AB, Bhosale DAV, Puttewar TY, Patil dry. design, development and evaluation of immediate release tablet of ibuprofen solid dispersion. Int $\mathrm{J}$ Curr Res. 2016;08:14.

[40] Panzade P. Pharmaceutical Cocrystal of Piroxicam: Design, Formulation and Evaluation. :10.

[41] Bandari S, Dronam VR, Eedara BB. Development and preliminary characterization of levofloxacin pharmaceutical cocrystals for dissolution rate enhancement. $\mathrm{J}$ Pharm Investig. 2017 Nov;47(6):583-91.

[42] Eddleston MD, Thakuria R, Aldous BJ, Jones W. An Investigation of the Causes of Cocrystal Dissociation at
High Humidity. J Pharm Sci. 2014 Sep;103(9):2859-64.

[43] Nechipadappu SK, Tekuri V, Trivedi DR. Pharmaceutical CoCrystal of Flufenamic Acid: Synthesis and Characterization of Two Novel Drug-Drug Co-Crystal. J Pharm Sci. 2017 May;106(5):1384 90.

[44] Seo J-W, Hwang K-M, Lee S-H, Kim D-W, Park E-S. Preparation and characterization of adefovir dipivoxil-stearic acid cocrystal with enhanced physicochemical properties. Pharm Dev Technol. 2018 Oct 21;23(9):890-9.

[45] Haneef J, Chadha R. Drug-Drug Multicomponent Solid Forms: Cocrystal, Coamorphous and Eutectic of Three Poorly Soluble Antihypertensive Drugs Using Mechanochemical Approach. AAPS PharmSciTech. 2017 Aug;18(6):2279-90.

[46] Hazarika JNR, deb p. formulation evaluation and optimization of immediate release tablet of aceclofenac by direct compression method. Int J Curr Pharm Res. 2017 May 5;9(3):118. 
[47] Otaki T, Tanabe Y, Kojima T, Miura M, Ikeda Y, Koide T, et al. In situ monitoring of cocrystals in formulation development using lowfrequency Raman spectroscopy. Int J Pharm. 2018 May;542(1-2):5665.

[48] Liu L, Zou D, Zhang Y, Zhang Q, Feng Y, Guo Y, et al. Pharmaceutical salts/cocrystals of enoxacin with dicarboxylic acids: Enhancing in vitro antibacterial activity of enoxacin by improving the solubility and permeability. Eur J Pharm Biopharm. 2020 Sep;154:62-73.

[49] Abidi SSA, Azim Y, Khan SN, Khan AU. Sulfaguanidine cocrystals: Synthesis, structural characterization and their antibacterial and hemolytic analysis. J Pharm Biomed Anal. 2018 Feb;149:351-7.

[50] Latif S, Abbas N, Hussain A, Arshad MS, Bukhari NI, Afzal H, et al. Development of paracetamolcaffeine co-crystals to improve compressional, formulation and in vivo performance. Drug Dev Ind Pharm. 2018 Jul 3;44(7):1099-108. 